\title{
Identification of Potential Prognostic Long Non-Coding RNA Biomarkers for Predicting Survival in Patients with Hepatocellular Carcinoma
}

\author{
Xiwen Liao ${ }^{a}$ Chengkun Yang ${ }^{a}$ Rui Huang ${ }^{b}$ Chuangye Han ${ }^{a}$ Tingdong Yu

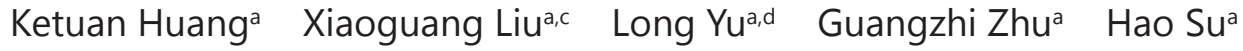 \\ Xiangkun Wang ${ }^{a}$ Wei Qin ${ }^{a}$ Jianlong Deng ${ }^{a, e} \quad X^{2}$ ianmin Zeng ${ }^{a} \quad$ Xinping Ye ${ }^{a}$ \\ Tao Penga
}

aDepartment of Hepatobiliary Surgery, the First Affiliated Hospital of Guangxi Medical University, Nanning, 'Department of Hematology, the First Affiliated Hospital of Guangxi Medical University, Nanning, 'Department of Hepatobiliary Surgery, Affiliated Hospital of Guangdong Medical University, Zhanjiang, dDepartment of Hepatobiliary and Pancreatic Surgery, the First Affiliated Hospital of Zhengzhou University, Zhengzhou, eDepartment of Hepatobiliary Surgery, the Sixth Affiliated Hospital of Guangxi Medical University, Yulin, China

\section{Key Words}

Lncrna • Hepatocellular carcinoma • Prognostic biomarker • TCGA • Bioinformatics analysis

\begin{abstract}
Background/Aims: The aim of the current study was to identify potential prognostic long non-coding RNA (IncRNA) biomarkers for predicting survival in patients with hepatocellular carcinoma (HCC) using The Cancer Genome Atlas (TCGA) dataset and bioinformatics analysis. Methods: RNA sequencing and clinical data of HCC patients from TCGA were used for prognostic association assessment by univariate Cox analysis. A prognostic signature was built using stepwise multivariable Cox analysis, and a comprehensive analysis was performed to evaluate its prognostic value. The prognostic signature was further evaluated by functional assessment and bioinformatics analysis. Results: Thirteen differentially expressed IncRNAs (DELs) were identified and used to construct a single prognostic signature. Patients with high risk scores showed a significantly increased risk of death (adjusted $P<0.0001$, adjusted hazard ratio $=3.522,95 \%$ confidence interval $=2.307-5.376$ ). In the time-dependent receiver operating characteristic analysis, the prognostic signature performed well for HCC survival prediction with an area under curve of $0.809,0.782$ and 0.79 for 1-, 3- and 5-year survival, respectively. Comprehensive survival analysis of the 13-DEL prognostic signature suggested that it serves as an independent factor in HCC, showing a better performance for prognosis prediction than
\end{abstract}


traditional clinical indicators. Functional assessment and bioinformatics analysis suggested that the prognostic signature was associated with the cell cycle and peroxisome proliferatoractivated receptor signaling pathway. Conclusions: The novel IncRNA expression signature identified in the present study may be a potential biomarker for predicting the prognosis of HCC patients.

\section{Introduction}

Liver cancer is prevalent in China and has become the fourth leading cause of male cancerrelated death [1]. The global cancer statistics demonstrate that the number of new cases and deaths from liver cancer in China account for more than half the worldwide total [2]. Most liver cancers are diagnosed as hepatocellular carcinoma (HCC) [3]. Hepatocarcinogenesis is driven by the interaction of genetic and environmental factors $[4,5]$. The main environmental factors associated with HCC are hepatitis B virus (HBV) infection, hepatitis C virus (HCV) infection and alcohol abuse [4]. Well-known genetic factors include the TP53 mutation, CTNNB1 mutation, methylation and dysregulation of cancer-relevant genes [4, 5]. The development of high-throughput technologies enabled researchers to investigate a variety of approaches for identifying the genetic factors associated with HCC through genome-wide screening, such as genome-wide association studies [6], genome-wide expression profiles [7] and comprehensive analysis of multiple genome-wide approaches [8].

Recent research advances support that the proportion of the genome termed noncoding RNAs (ncRNAs) reflects the complexity of the genome in higher eukaryotes $[9,10]$. Among ncRNAs, long non-coding RNA (lncRNA), have been studied extensively and shown to play indispensable roles in many biological processes, and increasing evidence suggests that dysregulated IncRNAs are associated with HCC tumorigenesis, metastasis, and prognosis [11-13].

A growing number of studies demonstrate that aberrantly expressed lncRNAs serve as potential biomarkers for predicting HCC prognosis $[14,15]$. Therefore, systematic efforts to investigate an expression-based lncRNA signature for prognosis prediction in patients with HCC are necessary. Genome-wide screening analysis is an effective method for the identification of the potential biomarkers for HCC diagnosis and prognosis prediction. The Cancer Genome Atlas (TCGA) is a project aimed at generating multi-dimensional maps of the key genomic changes in 33 types of cancer to discover potential genetic biomarkers for cancer prevention, diagnosis, and therapy based on comprehensive genomic data analysis [16]. The aim of the present study was to identify potential prognostic lncRNA biomarkers for predicting survival in patients with HCC using TCGA datasets and bioinformatics analysis.

\section{Materials and Methods}

\section{Data source}

An RNA sequencing (RNA-Seq) dataset of 371 HCC patients was obtained from TCGA data portal (https://portal.gdc.cancer.gov/, accessed November 5, 2017), including 374 HCC tumor tissues and 50 adjacent normal liver tissues [8]. The corresponding clinical information of TCGA HCC patients was download from the University of California Santa Cruz Xena browser (UCSC Xena: http://xena.ucsc.edu/, accessed November 5, 2017). Because the data were downloaded from TCGA, study approval by an ethics committee was not necessary. Data acquisition and application complied with TCGA publication guidelines and data access policies.

Screening of differentially expressed IncRNAs

Genome-wide IncRNA expression profiles were obtained from the RNA-Seq dataset of TCGA, which consists of mRNA and IncRNA expression data. LncRNAs with mean values of $>1$ were included in the differentially expressed lncRNA (DEL) screening. The edgeR [17] and DESeq [18] packages of the R platform 


\section{Cellular Physiology Cell Physiol Biochem 2018;48:1854-1869 \begin{tabular}{l|l|l} 
DOI: 10.1159/000492507 & and Biochemistry Published online: August 8, 2018 & $\begin{array}{l}\text { O } 2018 \text { The Author(s). Published by S. Karger AG, Basel } \\
\text { www.karger.com/cpb }\end{array}$ \\
\cline { 2 - 2 }
\end{tabular}}

Liao et al.: Prognostic IncRNA Biomarkers in Patients with HCC

were used for DEL screening. A false discovery rate (FDR) $<0.05$ and $\mid \log 2$ fold change $(\log 2 \mathrm{FC}) \mid \geq 1$ were identified as DELs in the comparison of expression levels between HCC tumor and adjacent normal liver tissues. The overlapping DELs between edgeR and DESeq were used for further analysis.

\section{Construction of the DEL expression-based prognostic signature}

The survival information of one HCC patient from TCGA was unknown, therefore, 370 HCC patients were finally included in the survival analysis. A univariate Cox regression analysis was performed using the survival package to examine the association between the expression levels of DELs and the overall survival (OS) of patients with HCC. DELs with a $P$-value $<0.001$ were considered prognostic DELs with expression levels significantly associated with OS. Prognostic DELs with a $P$-value of $<0.001$ in the survival analysis were further assessed using the "step" function to select the optimal combination. The prognostic DELs selected by the "step" function were used for construction of the prognostic model. The relative contribution of these prognostic DELs in HCC survival prediction was evaluated by fitting them into a multivariate Cox regression analysis with OS as the dependent variable. A DEL expression-based prognostic risk score model was constructed using the linear combination of the expression levels of DELs with the multivariate Cox regression coefficient $(\beta)$ as the weight. The risk score formula was as follows: risk score $=$ expression of $\mathrm{DEL}_{1} \times \beta_{1} \mathrm{DEL}_{1}+$ expression of $\mathrm{DEL}_{2} \times \beta_{2} \mathrm{DEL}_{2}+\ldots$ expression of $\mathrm{DEL}_{n} \times \beta_{n} \mathrm{DEL}_{\mathrm{n}}$ [19-21]. This DEL prognostic model was used to divide HCC patients into high- and low-risk groups using the median score of the DEL expression-based risk scores. A time-dependent receiver operating characteristic (ROC) curve was generated using the survivalROC package in the R platform to evaluate the accuracy of the DEL expressionbased prognostic signature for predicting OS in HCC [19].

\section{Comprehensive analysis of the DEL expression-based prognostic signature}

The prognostic model was evaluated by performing a comprehensive analysis of the DEL expressionbased prognostic signature. The expression levels of the prognostic DELs were compared between the lowand high-risk groups. In addition, the predictive value of the risk score for clinical status was investigated. Stratified analysis and joint effect analysis were performed to investigate the association between the risk score and clinical status in HCC.

\section{Functional assessment and bioinformatics analysis}

Generally, lncRNAs do not encode proteins, and their function is associated with coexpressed proteincoding genes (PCGs) $[22,23]$. The coexpression relationship between prognostic DELs and PCGs was evaluated using Pearson's correlation coefficient. Coexpressed PCGs were subjected to functional enrichment analysis to predict the biological function of the prognostic DELs using the Database for Annotation, Visualization, and Integrated Discovery version 6.7 (DAVID v6.7, https://david.ncifcrf.gov/, accessed November 5, 2017), which is a widely used bioinformatics resource [24-26]. GO terms and KEGG pathways with a $P$-value $<0.05$ and enrichment score $>1.0$ were considered as significantly enriched functional annotations.

The mRNA expression data of the PCGs were obtained from the same TCGA HCC patients and the same RNA-Seq dataset. Moreover, data processing of the PCG mRNA dataset was performed in the same manner as that of the IncRNA dataset, which was normalized by DESeq packages in the R platform. Prognostic DELs and PCGs with a Pearson correlation coefficient of $>0.4$ were considered lncRNA-correlated PCGs, and used for coexpression network construction and prognostic DEL functional assessment. A coexpression network between prognostic DELs and the corresponding PCGs was constructed using Cytoscape software (version 3.4.0; http://www.cytoscape.org/, accessed November 5, 2017) [27].

Gene set enrichment analysis (GSEA v.2-2.2.3: http://software.broadinstitute.org/gsea/index.jsp; accessed November 5,2017 ) was performed to investigate the potential mechanisms underlying the effect of the risk score on HCC prognosis $[28,29]$. The Molecular Signatures Database (MSigDB) of c2 (c2.cp.kegg. v6.1.symbols.gmt) and c5 (c5.all.v6.1.symbols.gmt) was used to assess the functional differences between the low- and high-risk groups [30]. The enrichment gene sets in the GSEA that reached a nominal $P$-value $<0.05$ and FDR $<0.25$ were considered significant. 


\section{Cellular Physiology Cell Physiol Biochem 2018;48:1854-1869 \begin{tabular}{ll|l} 
and Biochemistry & Dublished online: August 8, 2018 & $\begin{array}{l}\text { (c) } 2018 \text { The Author(s). Published by S. Karger AG, Basel } \\
\text { www.karger.com/cpb }\end{array}$
\end{tabular}

Statistical analysis

FDRs in edgeR, DESeq, and GSEA were adjusted for multiple testing with the Benjamini-Hochberg procedure to control FDR [31-33]. Univariate analysis of clinical features and OS was performed using the log-rank test; those clinical features with $P<0.05$ were included in the multivariate Cox proportional hazards regression model for adjustment. Coexpression relationships between prognostic DELs and PCGs were assessed using the Pearson correlation coefficient. Volcano plots and heat maps were drawn by the ggplot2 package in the R platform. A $P$-value $<0.05$ was considered statistically significant. All statistical analyses were performed using SPSS version 20.0 (IBM Corporation, Armonk, NY, USA) and R 3.3.0.

\section{Results}

\section{DEL screening}

Comprehensive gene annotation was downloaded from the Ensembl Genomes (Homo_sapiens.GRCh38.90.gtf: http:// www.ensembl.org/index.html, accessed November 5, 2017) [34, 35]. Of the 14, 447 lncRNAs obtained from the RNA-Seq dataset, 6, 938 lncRNAs met the criterion of a mean value $>1$ and were included in the DEL screening. In the present study, 2626 lncRNAs were identified as DELs using edgeR (Fig. 1A, Table S1), whereas, 842 IncRNAs were identified as DELs using DESeq (Fig. 1B, Table S2). For all supplemental material see www.karger. com/doi/10.1159/000492507. The 827 DELs that overlapped in the edgeR and DESeq screening were used for further analysis (Fig. 1C). The heat map of the 827 DELs is shown in Fig. 2.

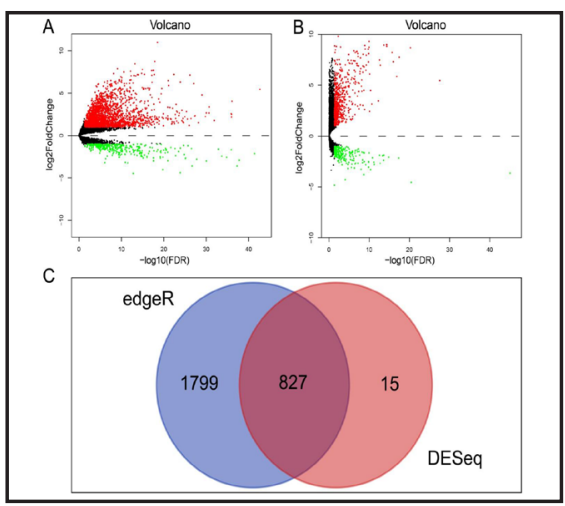

Fig. 1. Analysis of differentially expressed lncRNAs (DELs). (A) DELs identified using the edgeR package; (B) DELs identified using the DESeq package; (C) Overlapping DELs.

Table 1. The detailed information of 13 prognostic DELs and prognosis analysis in patients with

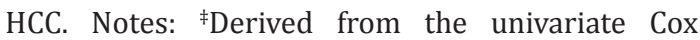
proportional hazards regression analysis in 370 HCC patients cohort; ${ }^{\&}$ Derived from the multivariate Cox proportional hazards regression analysis in 370 HCC patients cohort. Abbreviations: DEL, differentially expressed lncRNAs; HCC, hepatocellular carcinoma.

\begin{tabular}{lcccc}
\hline Ensemble ID & Gene name & Hazard ratio $^{\ddagger}$ & $P$-value ${ }^{\ddagger}$ & Coefficient\& $^{\&}$ \\
\hline ENSG00000264016 & AC015908.3 & 0.778685 & $1.02 \mathrm{E}-06$ & -0.1935 \\
ENSG00000237424 & FOXD2-AS1 & 1.264965 & 0.000723 & 0.1969 \\
ENSG00000267751 & AC009005.1 & 1.304236 & $3.79 \mathrm{E}-05$ & 0.1459 \\
ENSG00000251165 & F11-AS1 & 0.854905 & 0.000398 & 0.1081 \\
ENSG00000230587 & AC093609.1 & 0.798914 & 0.000701 & -0.1774 \\
ENSG00000271270 & TMCC1-AS1 & 1.561787 & $6.16 \mathrm{E}-08$ & 0.3089 \\
ENSG00000257732 & AC089983.1 & 1.284594 & 0.000198 & 0.1706 \\
ENSG00000227195 & MIR663AHG & 1.129568 & 0.000696 & -0.0803 \\
ENSG00000254290 & AC124067.4 & 1.119317 & 0.000746 & 0.1108 \\
ENSG00000276399 & FLJ36000 & 1.229365 & $8.22 \mathrm{E}-05$ & 0.1185 \\
ENSG00000235356 & AL592466.1 & 1.262443 & 0.000686 & -0.1715 \\
ENSG00000233571 & AL592043.1 & 1.434065 & $1.17 \mathrm{E}-06$ & 0.1593 \\
ENSG00000248762 & AC012339.1 & 1.342991 & 0.000219 & 0.1932 \\
\hline
\end{tabular}

\section{Construction of the DEL-based prognostic signature}

A univariate Cox regression analysis was performed to examine the association between DELs and the OS of patients with HCC. The results are shown in Table S3. Fortyseven DELs with a $P$-value $<0.001$ were regarded as prognostic DELs and selected using the "step" function to screen for the

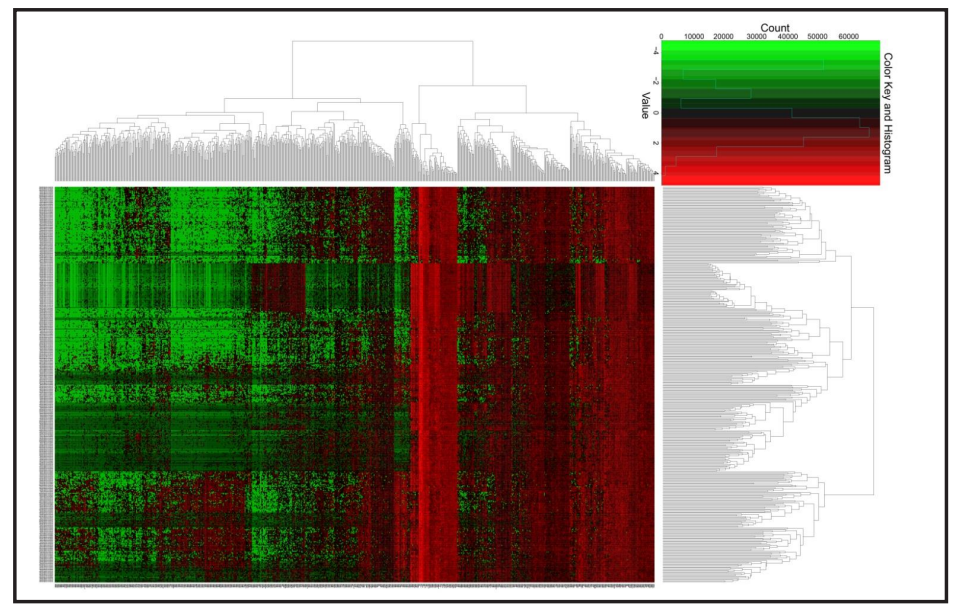

Fig. 2. Heat map of 827 DELs in HCC. 
optimal combination. The following 13 prognostic DELs were used for the prognostic model construction (summarized in Table 1): AC015908.3, FOXD2-AS1, AC009005.1, F11-AS1, AC093609.1, TMCC1-AS1, AC089983.1, MIR663AHG, AC124067.4, FLJ36000, AL592466.1, AL592043.1, and AC012339.1. Kaplan-Meier curves and a scatter plot of the 13 prognostic DELs are shown in Fig. $3 A-M$ and Fig. $3 N$, respectively. The relative contribution of these prognostic DELs to survival prediction was determined using multivariate Cox regression analysis. The risk score formula was as follows: risk score $=$ expression of AC015908.3 $\times(-0.1935)$

+ expression of FOXD2-AS1 $\times(0.1969)$

+ expression of AC009005.1 $\times(0.1459)$

+ expression of F11-AS1 $\times(0.1081)+$ expression of AC093609.1 $\times(-0.1774)+$ expression of TMCC1-AS1 $\times(0.3089)+$ expression of AC089983.1 $\times(0.1706)+$ expression of MIR663AHG $\times(-0.0803)+$ expression of AC124067.4 $\times(0.1108)$ + expression of FLJ36000 $\times(0.1185)+$ expression of AL592466.1 $\times(-0.1715)$ + expression of AL592043.1 $\times(0.1593)$ + expression of AC012339.1 × (0.1932). The results of the survival analysis of clinical features are summarized in Table 2 . Tumor stage and radical resection that were significantly associated with HCC OS in the univariate analysis were included in the multivariate Cox proportional hazards regression model for adjustment. Survival analysis indicated that the 13-DEL prognostic signature could separate HCC patients into low-risk and high-risk groups, and HCC patients with high-risk scores had

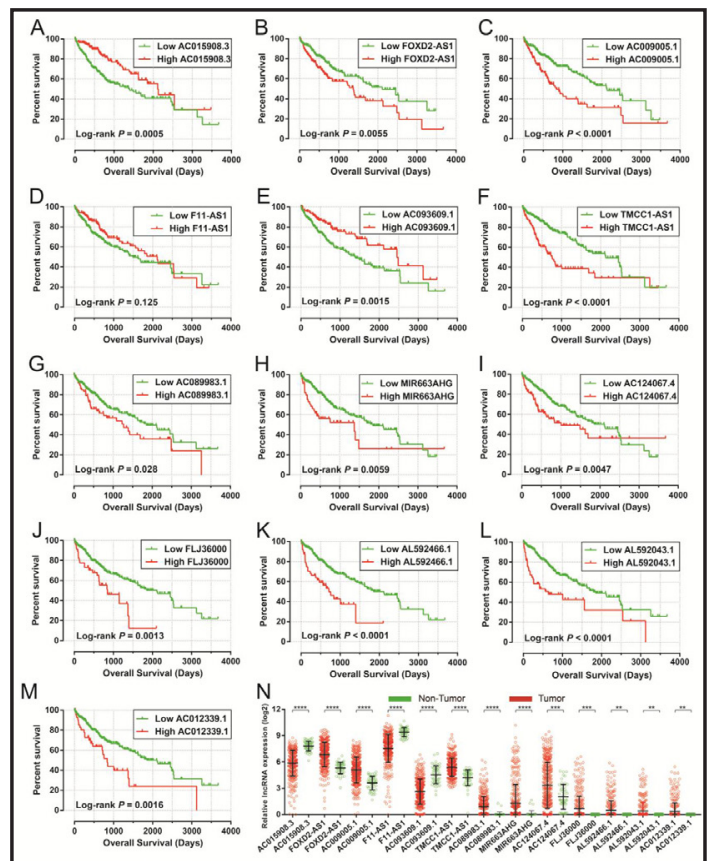

Fig. 3. Kaplan-Meier curves of overall survival (OS) of 13 prognostic DELs in HCC and their expression in HCC tumor tissues and adjacent normal liver tissues. Notes: The order of Kaplan-Meier curves of 13 prognostic DELs were as follow: (A) AC015908.3, (B) FOXD2-AS1, (C) AC009005.1, (D) F11-AS1, (E) AC093609.1, (F) TMCC1-AS1, (G) AC089983.1, (H) MIR663AHG, (I) AC124067.4, (J) FLJ36000, (K) AL592466.1, (L) AL592043.1, (M) AC012339.1; (N) Scatter plot of 13 prognostic DELs in HCC tumor tissues and adjacent normal liver tissues; ${ }^{* *} \mathrm{P}<0.01$, *** $\mathrm{P}<0.001$, **** $\mathrm{P}<0.0001$.

Fig. 4. Prognostic risk score model analysis of 13 prognostic DELs in HCC patients and their expression level in low- and high-risk groups. Notes: (A) From top to bottom are the risk score, patients' survival status distribution, and the expression heat map of 13 prognostic DELs in the low- and high-risk groups. (B) Kaplan-Meier curves for the low- and high-risk groups. (C) ROC curve analysis for predicting survival in HCC patients according to the risk score. (D) Scatter plot of the expression of 13 prognostic DELs expression level in the low- and high-risk groups. ${ }^{* * * *}$ $P<0.0001$.

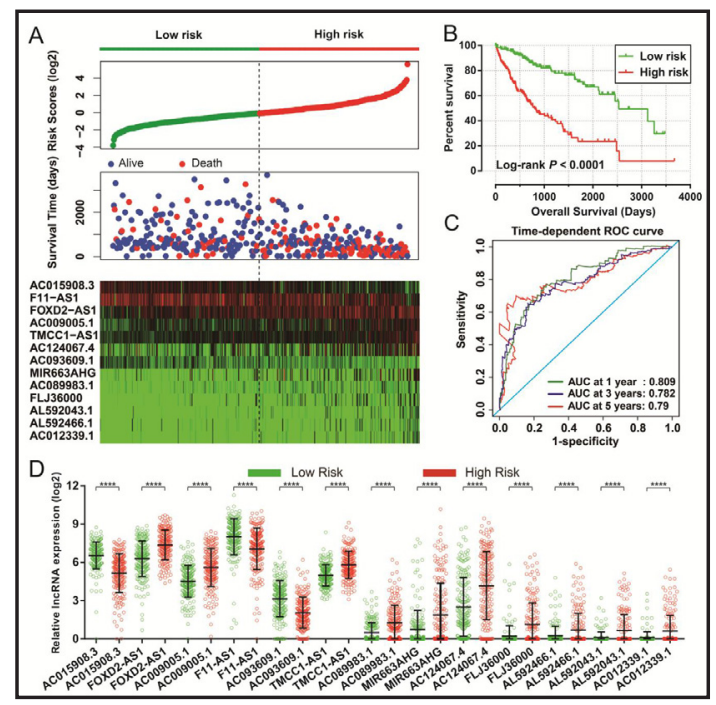


a significantly shorter median survival time (MST) than those with a low-risk score (802 vs. 2532 days for high-risk vs. low-risk, Table 2, Fig. $4 A, B$ ), and significantly increased risk of death (adjusted $P<0.0001$, adjusted hazard ratio $[\mathrm{HR}]=3.522,95 \%$ confidence interval $[\mathrm{CI}]=2.307-5.376$ for OS), after adjusting for tumor stage and radical resection. Time-dependent ROC analysis showed that the risk score performed well for HCC OS prediction, as the AUC of the time-dependent ROC curve was $0.809,0.782$ and 0.79 for 1-, 3- and 5-year survival prediction (Fig. 4C), respectively. The expression level distribution of the 13 prognostic DELs in the low- and high-risk groups are presented in Fig. 4D. In addition, we investigated the relationship between the 13-DEL prognostic signature and various clinical features (Fig. $5 A-G$ ), and the risk score showed a potential predictive value for predicting the status of tumor stage $(P=0.003$, AUC $=0.605,95 \% \mathrm{CI}=$ 0.535-0.676; Fig. 5B), histologic grade $(P<$ $0.0001, \mathrm{AUC}=0.642,95 \% \mathrm{CI}=0.585-0.700$; Fig. 5C), AFP $(P=0.002, \mathrm{AUC}=0.628,95 \%$ $\mathrm{CI}=0.550-0.707 ;$ Fig. $5 D$ ), radical resection $(P=0.007$, AUC $=0.630,95 \% \mathrm{CI}=0.545-$ $0.715 ;$ Fig. $5 E$ ) and micro vascular invasion $(P=0.008$, AUC $=0.591,95 \% \mathrm{CI}=0.524-$ 0.657; Fig. 5F).

\section{Stratified and joint effects analysis}

To investigate the prognostic prediction value of the 13-DEL prognostic signature under different clinical conditions, stratified and joint effects analyses were performed after adjusting for tumor stage and radical resection. In the stratified analysis, high-risk scores predicted a significantly increased risk of death in patients with all favorable strata and all adverse strata except in patients without radical resection and serum AFP $>400 \mathrm{ng} /$ $\mathrm{mL}$ (Fig. 6A), indicating that the risk score is independent from clinical information. To facilitate the use of the risk score, a survival nomogram was plotted (Fig. 6B) based on 155 patients with complete clinical information and considering the risk scores and the clinicopathological observations. Consistent with the survival analysis, the risk score contributed the greatest number of risk points (ranged 0-100), whereas the other clinical information had a considerably lower
Table 2. Distribution of HCC patients' characteristics and prognosis analysis. Notes: a Information of alcohol consumption was unavailable in 19 patients; ${ }^{\mathrm{b}}$ Information of ishak fibrosis score was unavailable

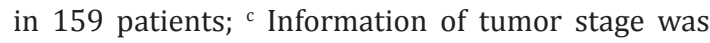
unavailable in 24 patients; ${ }^{\mathrm{d}}$ Information of histologic grade was unavailable in 5 patients; ${ }^{\mathrm{e}}$ Information of serum AFP was unavailable in 93 patients; ${ }^{f}$ Information of radical resection was unavailable in 7 patients; ${ }^{g}$ Information of micro vascular invasion was unavailable in 56 patients; ${ }^{\mathrm{h}}$ Information of Child-Pugh score was unavailable in 132 patients; Abbreviations: HCC, hepatocellular carcinoma; MST, median survival time; HR, hazard ratio; CI, confidence interval; AFP, $\alpha$-fetoprotein; MVI, micro vascular invasion.

\begin{tabular}{|c|c|c|c|c|}
\hline Variables & Events/total(n=370) & MST (days) & HR (95\% Cl) & Log-rank $P$ \\
\hline Age (years) & & & & 0.194 \\
\hline$\leq 65$ & $73 / 232$ & 2465 & 1 & \\
\hline$>65$ & $57 / 138$ & 1423 & $1.265(0.893-1.791)$ & \\
\hline Sex & & & & 0.261 \\
\hline female & $51 / 121$ & 1490 & 1 & \\
\hline male & $79 / 249$ & 2486 & $0.817(0.573-1.164)$ & \\
\hline Alcohol consumption a & & & & 0.896 \\
\hline oo & $84 / 234$ & 1694 & 1 & \\
\hline YES & $40 / 117$ & 1624 & $1.026(0.703-1.496)$ & \\
\hline Ishak fibrosis score b & & & & 0.872 \\
\hline 0 - No Fibrosis & $30 / 74$ & 2131 & 1 & \\
\hline 1,2 - Portal Fibrosis & 9/31 & 1372 & $0.917(0.429-1.962)$ & \\
\hline 3,4-Fibrous Speta & $6 / 28$ & $\mathrm{NA}$ & $0.682(0.281-1.654)$ & \\
\hline 5 - Nodular Formation and Incomplete Cirrhosis & 2/9 & 1386 & $0.750(0.177-3.167)$ & \\
\hline 6-Established Cirrhosis & $17 / 69$ & $\mathrm{NA}$ & $0.766(0.418-1.403)$ & \\
\hline Tumor stage c & & & & $<0.0001$ \\
\hline I & $42 / 171$ & 2532 & 1 & \\
\hline II & $26 / 85$ & 1852 & $1.427(0.874-2.330)$ & \\
\hline $\mathrm{III+IV}$ & $48 / 90$ & 770 & $2.764(1.823-4.190)$ & \\
\hline Histologic grade ${ }^{d}$ & & & & 0.748 \\
\hline G1 & $18 / 55$ & 2116 & 1 & \\
\hline G2 & $60 / 177$ & 1685 & $1.181(0.697-2.000)$ & \\
\hline G3 & $43 / 121$ & 1622 & $1.233(0.711-2.140)$ & \\
\hline G4 & $5 / 12$ & $\mathrm{NA}$ & $1.693(0.626-4.584)$ & \\
\hline $\operatorname{Serum~AFP~}(\mathrm{ng} / \mathrm{mL})^{\mathrm{e}}$ & & & & 0.832 \\
\hline$\leq 400$ & $62 / 213$ & 2456 & 1 & \\
\hline$>400$ & $22 / 64$ & 2486 & $1.055(0.645-1.724)$ & \\
\hline Radical resection ${ }^{f}$ & & & & 0.006 \\
\hline Ro & $110 / 323$ & 1852 & 1 & \\
\hline $\mathrm{R} 1+\mathrm{R} 2+\mathrm{RX}$ & $17 / 40$ & 837 & $2.030(1.213-3.395)$ & \\
\hline MVI 8 & & & & 0.154 \\
\hline № & $60 / 206$ & 2131 & 1 & \\
\hline YES & $36 / 108$ & 2486 & $1.351(0.892-2.047)$ & \\
\hline Child-Pugh score h & & & & 0.18 \\
\hline A & $59 / 216$ & 2542 & 1 & \\
\hline$B+C$ & 9/22 & 1005 & $1.614(0.796-3.270)$ & \\
\hline Risk score & & & & $<0.0001$ \\
\hline Low & $39 / 185$ & 2532 & 1 & \\
\hline High & $81 / 185$ & 802 & $3.929(2.678-5.765)$ & \\
\hline
\end{tabular}


Table 3. Joint effects survival analysis of clinical factors and the DELs signature risk score with OS in HCC patients. Notes: ${ }^{\mathrm{b}}$ Information of ishak fibrosis score was unavailable in 159 patients; ${ }^{\mathrm{c}}$ Information of tumor stage was unavailable in 24 patients; ${ }^{\mathrm{d}}$ Information of histologic grade was unavailable in 5 patients; ${ }^{\mathrm{e}}$ Information of serum AFP was unavailable in 93 patients; ${ }^{\mathrm{f}}$ Information of radical resection was unavailable in 7 patients; ${ }^{\mathrm{g}}$ Information of micro vascular invasion was unavailable in 56 patients; ${ }^{\mathrm{h}}$ Information of ChildPugh score was unavailable in 132 patients; £Adjusted for tumor stage and radical resection. Abbreviations: DEL, differentially expressed IncRNA; OS, overall survival; HCC, hepatocellular carcinoma; MST, median survival time; HR, hazard ratio; CI, confidence interval; AFP, $\alpha$-fetoprotein; MVI, micro vascular invasion.

\begin{tabular}{|c|c|c|c|c|c|c|c|c|}
\hline Group & Risk Score & Variables & Events/total $(\mathrm{n}=370)$ & MST (days) & Crude HR $(95 \%$ CI) & Crude $P$ & Adjusted HR $(95 \% \mathrm{CI})$ & Adjusted $P E$ \\
\hline & & Ishak fibrosis score & & & & & & \\
\hline 1 & Low risk & 0 & $13 / 47$ & 3125 & 1 & & 1 & \\
\hline 2 & Low risk & $1 / 2 / 3 / 4 / 5 / 6$ & $13 / 77$ & NA & $0.842(0.374-1.895)$ & 0.678 & $0.935(0.398-2.196)$ & 0.877 \\
\hline 3 & High risk & 0 & $17 / 27$ & 660 & $4.037(1.871-8.711)$ & 0.0004 & $3.584(1.600-8.030)$ & 0.002 \\
\hline \multirow[t]{2}{*}{4} & High risk & $1 / 2 / 3 / 4 / 5 / 6$ & $21 / 60$ & 1372 & $2.870(1.348-6.112)$ & 0.006 & $3.235(1.465-7.144)$ & 0.004 \\
\hline & & Tumor Stage c & & & & & & \\
\hline A & Low risk & Stage I & $14 / 100$ & NA & 1 & & 1 & \\
\hline B & Low risk & Stage II & $6 / 38$ & 3258 & $1.176(0.451-3.0650$ & 0.74 & $1.174(0.450-3.062)$ & 0.743 \\
\hline $\mathrm{C}$ & Low risk & Stage III+IV & $13 / 34$ & 1622 & $3.463(1.625-7.381)$ & 0.001 & $3.431(1.609-7.316)$ & 0.001 \\
\hline $\mathrm{D}$ & High risk & Stage I & $28 / 71$ & 1372 & $4.701(2.451-9.017)$ & $<0.0001$ & $4.643(2.416-8.923)$ & $<0.0001$ \\
\hline E & High risk & Stage II & $20 / 47$ & 848 & $5.724(2.868-11.427)$ & $<0.0001$ & $5.661(2.833-11.310)$ & $<0.0001$ \\
\hline \multirow[t]{2}{*}{$\mathrm{F}$} & High risk & Stage III+IV & $35 / 56$ & 419 & $7.954(4.254-14.872)$ & $<0.0001$ & $7.300(3.852-13.832)$ & $<0.0001$ \\
\hline & & Histologic Grade ${ }^{d}$ & & & & & & \\
\hline $\mathrm{a}$ & Low risk & G1 & $8 / 37$ & 2131 & 1 & & 1 & \\
\hline $\mathrm{b}$ & Low risk & G2 & $18 / 95$ & 3125 & $0.900(0.391-2.073)$ & 0.804 & $1.189(0.437-3.237)$ & 0.735 \\
\hline $\mathrm{c}$ & Low risk & $\mathrm{G} 3+\mathrm{G} 4$ & $10 / 49$ & NA & $0.885(0.348-2.250)$ & 0.798 & $1.256(0.428-3.686)$ & 0.678 \\
\hline d & High risk & G1 & $10 / 18$ & 581 & $3.514(1.372-9.005)$ & 0.009 & $3.764(1.198-11.833)$ & 0.023 \\
\hline e & High risk & G2 & $42 / 82$ & 802 & $4.087(1.912-8.737)$ & 0.0003 & $4.459(1.734-11.467)$ & 0.002 \\
\hline \multirow[t]{2}{*}{ f } & High risk & $\mathrm{G} 3+\mathrm{G} 4$ & $38 / 84$ & 899 & $3.461(1.606-7.458)$ & 0.002 & $3.920(1.523-10.088)$ & 0.005 \\
\hline & & Serum AFP $(\mathrm{ng} / \mathrm{mL})$ & & & & & & \\
\hline I & Low risk & $\leq 400$ & $20 / 121$ & 2532 & 1 & & 1 & \\
\hline II & Low risk & $>400$ & $6 / 26$ & NA & $1.112(0.443-2.790)$ & 0.821 & $1.206(0.478-3.040)$ & 0.691 \\
\hline III & High risk & $\leq 400$ & $42 / 92$ & 1149 & $4.113(2.397-7.056)$ & $<0.0001$ & $3.978(2.269-6.976)$ & $<0.0001$ \\
\hline \multirow[t]{2}{*}{ IV } & High risk & $>400$ & $16 / 38$ & 2486 & $3.217(1.659-6.237)$ & 0.001 & $2.671(1.301-5.481)$ & 0.007 \\
\hline & & Radical resection ${ }^{f}$ & & & & & & \\
\hline 11 & Low risk & R0 & $33 / 169$ & 3125 & 1 & & 1 & \\
\hline 22 & Low risk & $\mathrm{R} 1+\mathrm{R} 2+\mathrm{RX}$ & $6 / 14$ & 837 & $4.207(1.752-10.103)$ & 0.001 & $3.739(1.300-10.753)$ & 0.014 \\
\hline 33 & High risk & R0 & $77 / 154$ & 802 & $4.337(2.860-6.579)$ & $<0.0001$ & $3.991(2.557-6.230)$ & $<0.0001$ \\
\hline \multirow[t]{2}{*}{44} & High risk & $\mathrm{R} 1+\mathrm{R} 2+\mathrm{RX}$ & $11 / 26$ & 837 & $4.820(2.418-9.607)$ & $<0.0001$ & $3.692(1.719-7.929)$ & 0.01 \\
\hline & & MVIg & & & & & & \\
\hline $\mathrm{i}$ & Low risk & NO & $23 / 119$ & 3125 & 1 & & 1 & \\
\hline ii & Low risk & YES & $11 / 50$ & 3258 & $1.364(0.663-2.806)$ & 0.399 & $1.104(0.470-2.595)$ & 0.82 \\
\hline iii & High risk & NO & $37 / 87$ & 1372 & $3.853(2.248-6.603)$ & $<0.0001$ & $3.608(2.019-6.447)$ & $<0.0001$ \\
\hline \multirow[t]{2}{*}{ iv } & High risk & YES & $25 / 58$ & 1149 & $4.144(2.317-7.411)$ & $<0.0001$ & $3.399(1.697-6.807)$ & 0.001 \\
\hline & & Child-Pugh score ${ }^{h}$ & & & & & & \\
\hline (1) & Low risk & A & $21 / 116$ & 3258 & 1 & & 1 & \\
\hline (2) & Low risk & $\mathrm{B}+\mathrm{C}$ & $3 / 13$ & NA & $1.188(0.351-4.024)$ & 0.782 & $1.240(0.366-4.198)$ & 0.729 \\
\hline (3) & High risk & A & $38 / 100$ & 1386 & $3.272(1.883-5.6860$ & $<0.0001$ & $3.098(1.771-5.420)$ & $<0.0001$ \\
\hline (4) & High risk & $\mathrm{B}+\mathrm{C}$ & $6 / 9$ & 535 & $9.017(3.530-23.034)$ & $<0.0001$ & $7.111(2.747-18.408)$ & $<0.0001$ \\
\hline
\end{tabular}

contribution.

Joint effects analysis of the 13-DEL prognostic signature and clinical features indicated that the prognostic signature performed well in clinical outcome prediction, and in combination with clinical features, it showed a strong interaction and better predictive value for HCC prognosis (Fig. 7A-G and Table 3).

\section{Functional assessment and bioinformatics analysis}

A total of 17, 682 PCGs were extracted from the TCGA HCC patients' dataset and used for coexpression network construction. The Pearson correlation coefficients of 686 
Fig. 5. The predictive value of the risk score for HCC clinical parameters. Notes: ROC curve of risk score for clinical parameters: (A) Ishak fibrosis score, (B) tumor stage, (C) histologic grade, (D) serum AFP, (E) radical resection, (F) micro vascular invasion, and (G) ChildPugh score.

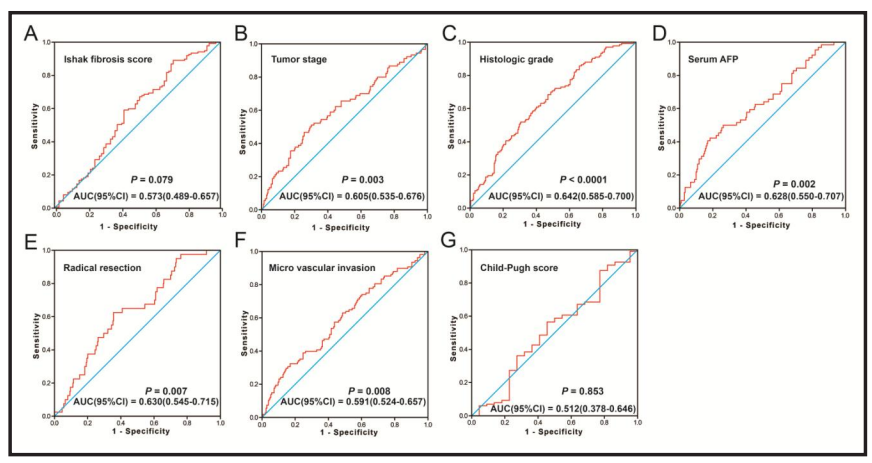

Fig. 6. The relationship between risk score and clinical information. Notes: (A) Stratified analysis of the association between risk score and OS in HCC. (B) Nomogram for predicting the 1-,3- and 5 -year event (death) with risk score and clinical information.

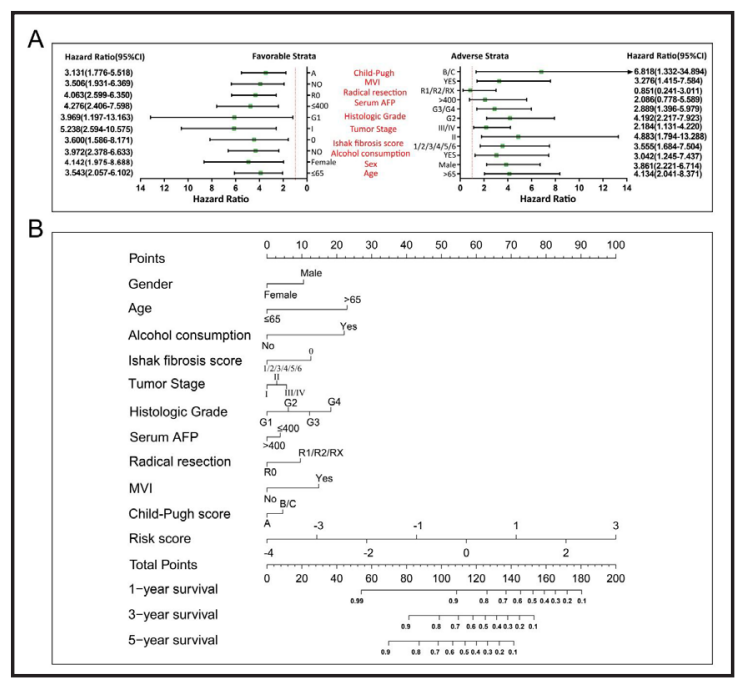

Fig. 7. Joint effects analysis of OS stratified by risk score and HCC clinical parameters. Notes: Joint effects analysis stratified by risk score and following clinical parameters: (A) Ishak fibrosis score, (B) tumor stage, (C) histologic grade, (D) serum AFP, (E) radical resection, (F) micro vascular invasion; (G) Child-Pugh score.

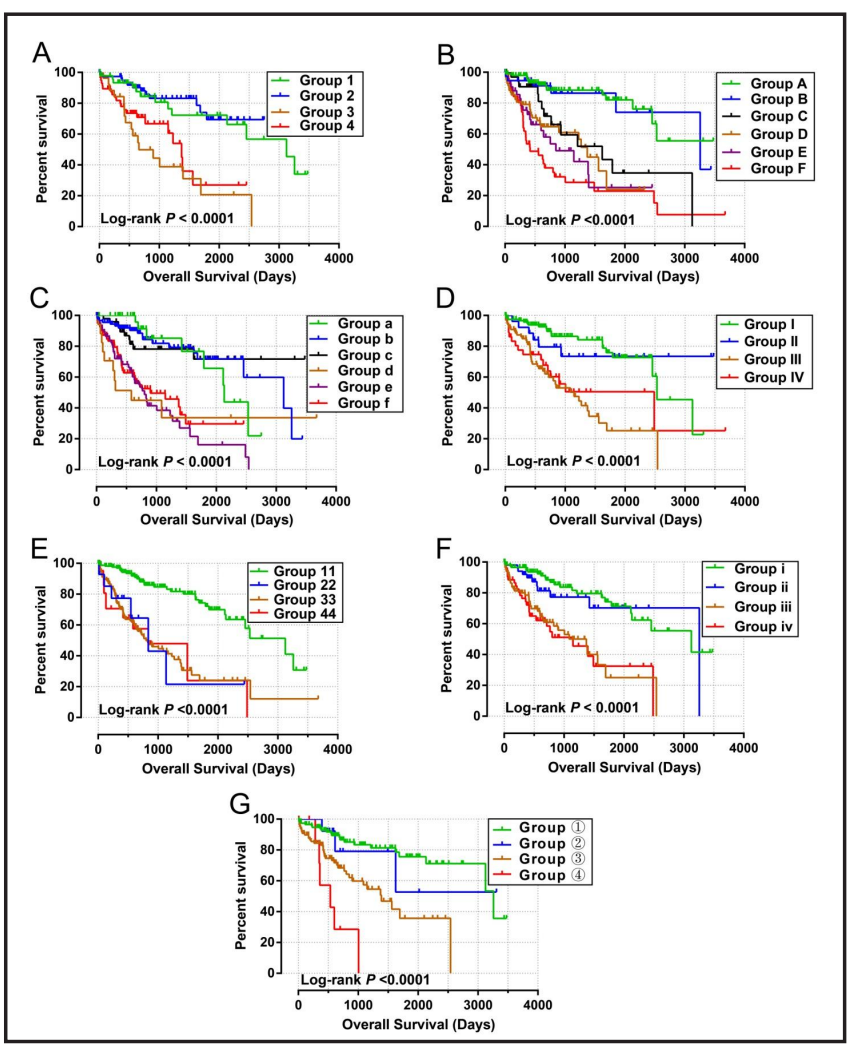




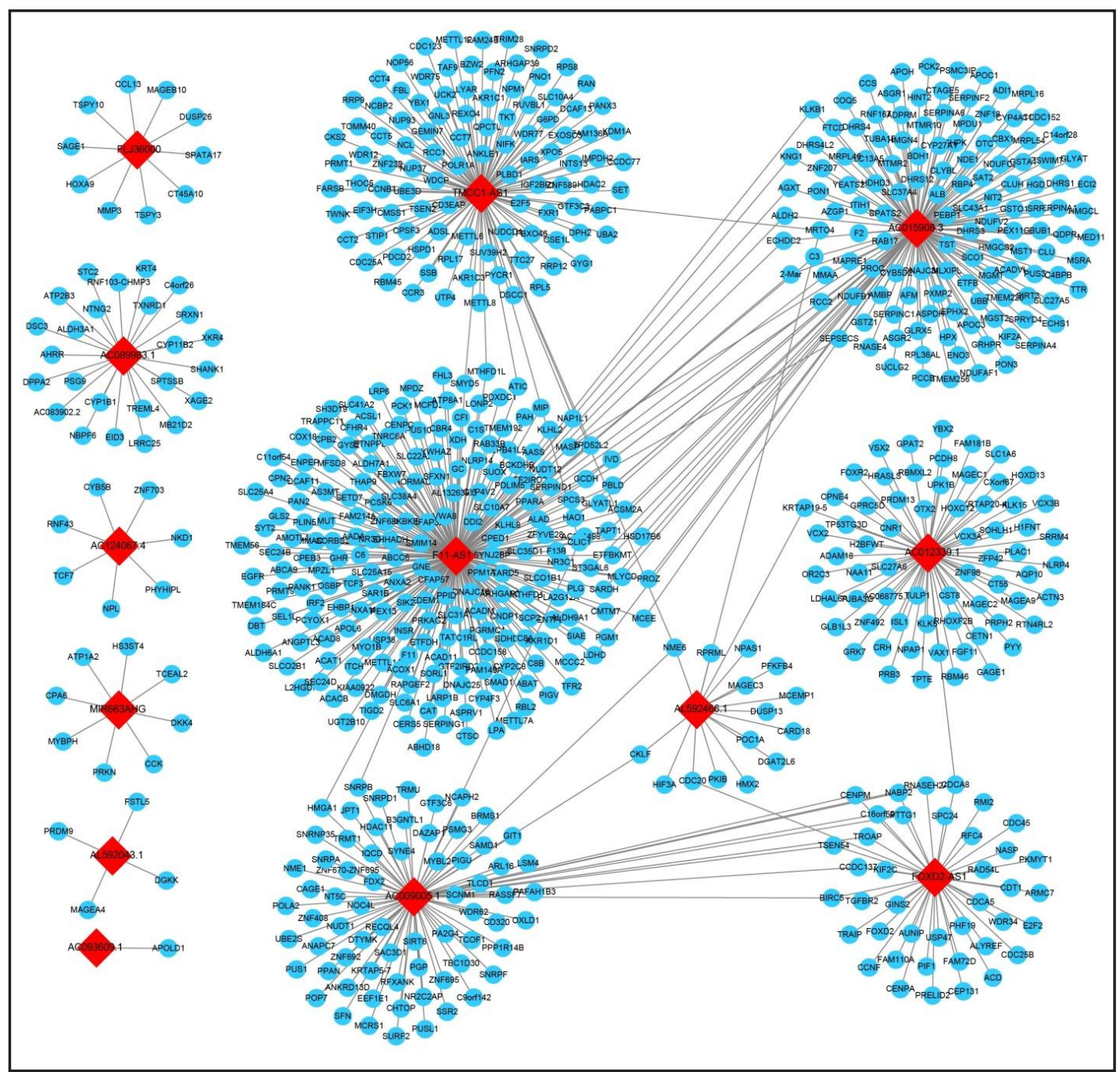

Fig. 8. Co-expression network of 13 prognostic DELs and corresponding lncRNA-correlated PCGs.

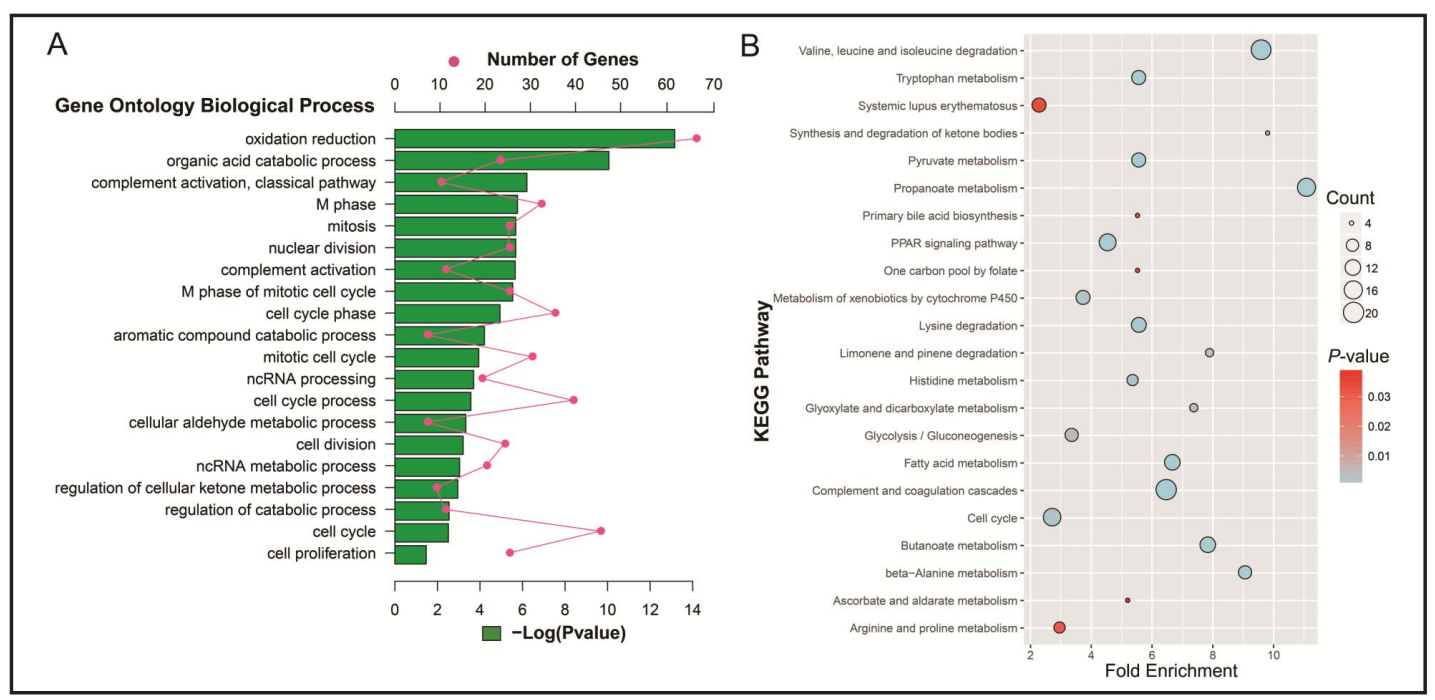

Fig. 9. Functional assessment of IncRNA-correlated PCGs of 13 prognostic DELs. Notes: (A) GO term enrichment results. (B) KEGG enrichment results. 
lncRNA-correlated PCGs with 738 edges greater than 0.4 were included in the coexpression network (Fig. 8 and Table S4). To investigate the potential functional roles of these 13 prognostic lncRNAs in HCC, we performed functional enrichment analysis of IncRNA-correlated PCGs using DAVID v6.7. GO term enrichment analysis suggested that these IncRNA-correlated PCGs were significantly enriched in 273 GO terms (Table S5), of which cell cycle-related

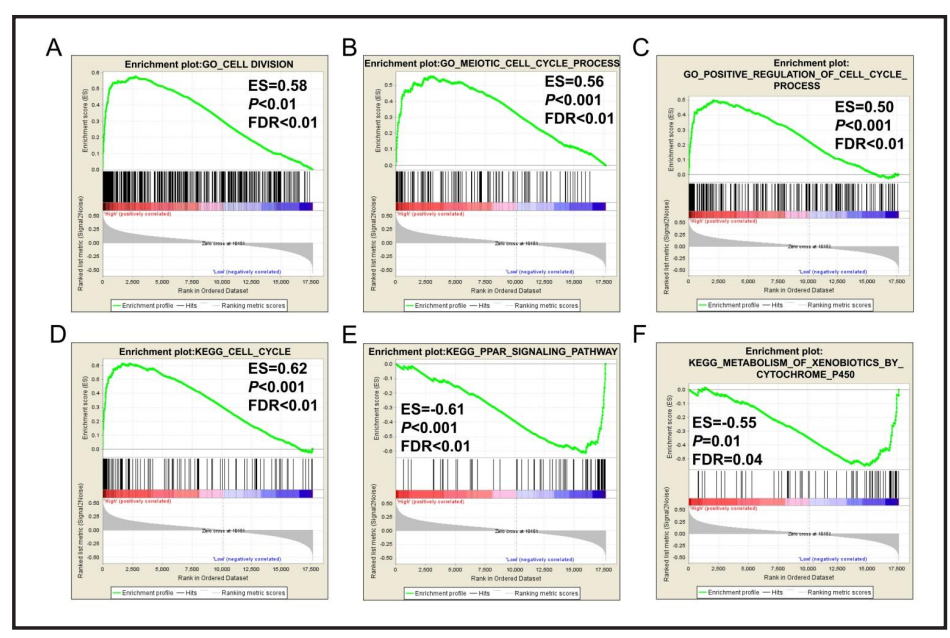

Fig. 10. GSEA results of $c 2$ and $c 5$ MSigDB between low- and high-risk groups. Notes: GSEA results of c2 MSigDB (A-C) and c5 MSigDB(D-F). biological processes were frequently enriched (Fig. 9A). Consistent with the GO enrichment analysis, KEGG enrichment analysis confirmed that these IncRNA-correlated PCGs were significantly enriched in the cell cycle pathway. Additionally, the peroxisome proliferator-activated receptor (PPAR) signaling pathway and multiple metabolic pathways were also enriched, including glyoxylate and dicarboxylate metabolism, metabolism of xenobiotics by cytochrome P450, beta-alanine metabolism, and propanoate metabolism (Fig. 9B).

GSEA analysis of the low- and high-risk groups supported the results of functional enrichment analysis for IncRNA-correlated PCGs. Cell cycle-related biological processes and pathways, and the PPAR signaling pathway were significantly enriched (Table S6 and S7; Fig. $10 A-F$ ), indicating that the 13-DEL prognostic signature may have a marked influence on these biological processes and pathways in HCC.

\section{Discussion}

TCGA is a comprehensive expression database of multiple types of cancer. Several recent studies used the IncRNA dataset from TCGA to investigate the potential use of IncRNAs for cancer diagnosis and prognosis prediction. He et al., Tang et al. and Zang et al. used TCGA lncRNA dataset to identify a lncRNA expression-based prognostic signature for assessing the survival of patients with soft-tissue sarcoma, lung cancer, and colorectal adenocarcinoma [36-38]. TCGA IncRNA datasets of cutaneous melanoma [39], bladder urothelial carcinoma [40], breast cancer [41], clear cell renal cell carcinoma [42], gastric cancer [43, 44], head and neck squamous cell carcinoma [45], and HCC [46] have been used in IncRNA expressionbased prognostic signature investigations by other researchers. These studies used a genome-wide screening method to identify potential prognostic lncRNAs and calculated a risk score based on IncRNA expression. This is an effective method to screen for prognostic lncRNAs and investigate their optimal combination for prognosis prediction.

Numerous studies by multiple researchers, who systematically and comprehensively reviewed the existing literature on the role of IncRNAs in HCC indicate that IncRNAs can be dysregulated in HCC and play a critical role in hepatocarcinogenesis, metastasis, as therapeutic targets, and in prognosis or diagnosis [14, 47, 48]. Yang et al. identified numerous recurrently deregulated tumorigenesis- and metastasis-associated lncRNAs, and most of these were experimentally validated and mechanistically linked to cancer development and progression [49]. Yuan et al. reported that a lncRNA associated with microvascular invasion in HCC (lncRNA MVIH) was frequently overexpressed in HCC patients with microvascular 
invasion, and deregulation of IncRNA MVIH serves as a predictor for poor OS and recurrencefree survival after hepatectomy [12]. Because lncRNAs play important roles in HCC, it is necessary to identify potential lncRNA biomarkers in HCC, and the use of TCGA HCC IncRNA dataset is a reliable method.

In the present study, we identified 827 DELs via the edgeR and DESeq packages, and identified 13 DELs that were suitable for the construction of a prognostic signature through screening with the "step" function. The DEL-based prognostic signature performed well in HCC prognosis prediction, and the time-dependent ROC analysis showed that it performed well in 1-, 3-, and 5-year survival prediction. In addition, the DEL-based prognostic signature identified in the present study performed well compared with the previous prognostic signature reported by Wang et al., and the prediction accuracy was significantly improved (AUC in 5-year survival prediction: 0.79 vs. 0.701, respectively) [46]. Consistent with previous findings, AC093609.1 and AC124067.4 were also identified as HCC prognostic DELs in the present analysis [46]. In addition, AC124067.4, also known as RP11-150012.3, was previously identified as a prognostic DEL in a TCGA lncRNA-based prognostic signature investigation in colorectal adenocarcinoma and gastric cancer $[38,44]$. A previous study showed that the expression levels of TMCC1-AS1, which was included in the 13-DEL prognostic signature, are associated with OS in patients with HCC [50]. Yu et al. reported that another prognostic DEL, FOXD2-AS1, was significantly differentially expressed in association with the progression of gastric cancer, and high FOXD2-AS1 expression was associated with a significantly increased risk of death [51]. Similar results regarding FOXD2-AS1 were reported in non-small cell lung cancer. Rong et al. showed that high expression of FOXD2-AS1 promotes non-small cell lung cancer progression via Wnt/ $\beta$-catenin signaling and is associated with poor OS [52]. A study in colorectal cancer (CRC) also suggested that FOXD2-AS1 was significantly upregulated in tumor tissues and acts as a tumor promoter in CRC, inducing CRC progression by regulating epithelial-mesenchymal transition and the Notch signaling pathway [53]. Transcriptional fingerprint analysis in pancreatic ductal adenocarcinoma (PDAC) demonstrated that F11AS1 is downregulated in PDAC tumor tissues and cell lines. These results suggest that dysregulated lncRNAs play important roles in PDAC development and progression [54]. A literature review revealed that the remaining prognostic DELs identified in the present study were not reported previously, and their function in HCC and other cancers needs to be further investigated and verified. The present findings have limitations because they were obtained from a single cohort; therefore, external validation cohorts are necessary to confirm the present results.

Survival analysis of the DEL-based prognostic signature in the present study showed a significantly different clinical outcome after stratification by risk score. Stratification according to risk score and clinical features suggest that the prognostic signature constructed in the current study may serve as an independent risk factor for HCC. Joint effects analysis of risk scores and clinical features suggested that the prognostic signature may have a strong interaction with clinical features and serve as an independent factor for HCC prognosis. The nomogram substantiated that the risk score contributed most to HCC prognosis prediction. However, because of the lack of validation cohorts, further studies are needed to confirm our results. Functional analysis of these prognostic DELs have rarely been reported in previous studies, therefore, the mechanism underlying the effect of these prognostic DELs on HCC prognosis still needs in-depth exploration.

Functional assessment of the HCC prognostic DELs in PCGs showed that cell cycle biological processes and pathways were significantly enriched. Previous genome-wide screening studies using IncRNA datasets from GEO and TCGA in lung cancer [55, 56], gastric cancer [44] and breast cancer [57, 58] also identified cell cycle-related lncRNAs. Several studies reported that dysregulation of specific lncRNAs in HCC cell lines caused cell cycle arrest and induced apoptosis, and affected the prognosis of HCC [59, 60]. KEGG enrichment analysis revealed a significant enrichment in the PPAR signaling pathway. Zhang et al. reported that the IncRNA HOTTIP is upregulated and has a high diagnostic value in HCC, and the genes coexpressed with HOTTIP are related to the PPAR signaling pathway [61]. In a genome-wide

\section{KARGER}


analysis of intrahepatic cholangiocarcinoma (iCCA), coexpressed mRNAs of DELs in iCCA tissues were significantly enriched in the PPAR signaling pathway, and KEGG enrichment of DEL target genes was demonstrated in papillary thyroid carcinoma $[62,63]$. The most enriched functional terms of the KEGG analysis in coexpressed PCGs were metabolism-related pathways, such as glyoxylate and dicarboxylate metabolism, and metabolism of xenobiotics by cytochrome P450. These metabolism-related pathways are associated with liver function and indicating that dysregulated DELs are involved the function of the liver and may play a role in hepatocarcinogenesis. GSEA of low- and high-risk groups demonstrated that cell cycle and PPAR signaling pathways were significantly enriched, and this was confirmed by the PCG enrichment results. We speculate that the prognostic signature identified in the present study may play a vital role in cell cycle and PPAR signaling pathway regulation in HCC. However, these findings need further experimental verification.

Although TCGA database was previously used to analyze lncRNA-related signatures for HCC prognosis, the present study was different in that it investigated the optimum combination of these prognostic IncRNAs using the "step" function in the R platform [46]. Therefore, the prognostic signature constructed in the present study is more accurate than that identified in a previous study [46]. In addition, comprehensive survival analysis of the present 13DEL prognostic signature was performed by developing a nomogram, and stratified and joint effects survival analysis was performed to investigate its potential application. The stratified and joint effects analysis of the risk score and clinical features suggested that the 13-DEL prognostic signature was an independent risk factor for HCC, and the associated risk score could serve as a prognostic indicator for HCC patients. The nomogram consisting of the risk score and other clinical information indicated that the contribution of the risk score to HCC prognosis prediction was greater than that of other traditional clinical indicators.

The present study had several limitations. First, the results generated in the present study were derived from a single cohort, and the demographics of the patients from TCGA may not be representative of all patient populations; therefore, the genetic alterations may be biased, and further validation in other HCC cohorts is necessary. Second, the clinical information from TCGA database was incomplete; therefore, we were unable to perform a comprehensive stratification analysis in all strata of HCC, as well as adjust all the features that may affect HCC OS in the Cox proportional hazard regression model.

Despite these limitations, the current study has identified numerous DELs with potential diagnostic and prognostic value for HCC. In addition, we constructed an effective prognostic signature to predict HCC prognosis that may be valuable for clinical application.

\section{Conclusion}

Here, we performed a genome-wide screening analysis using an HCC dataset and identified 827 DELs. We then constructed a prognostic signature based on the expression of 13 prognostic DELs. Comprehensive survival analysis of the 13-DEL prognostic signature suggested that the risk score of the prognostic signature serves as an independent factor in HCC and performs better for prognosis prediction than other traditional clinical indicators. The results suggest that the 13-DEL prognostic signature is a potential biomarker for the prognosis of HCC patients, and could be a clinically useful tool that can be easily incorporated into an RNA-Seq clinical sequencing program to individualize HCC therapy. Functional assessment and bioinformatics analysis showed that cell cycle-related processes and pathways and the PPAR signaling pathway were significantly associated with the risk score, indicating that the 13-DEL prognostic signature may be involved in the regulation of the status of HCC cells. However, further studies are necessary to elucidate the mechanism underlying the roles of these DELs in HCC, and additional cohorts are needed to validate our findings. Additional findings of the present study are also worthy of further investigation. 


\section{Cellular Physiology Cell Physiol Biochem 2018;48:1854-1869 \begin{tabular}{ll|l} 
DOI: 10.1159/000492507 & and Biochemistry Published online: August 8, 2018 & $\begin{array}{l}\text { O } 2018 \text { The Author(s). Published by S. Karger AG, Basel } \\
\text { www.karger.com/cpb }\end{array}$
\end{tabular}}

\section{Acknowledgements}

This work was supported in part by the National Nature Science Foundation of China (No.: 81560535, 81072321, 30760243, 30460143 and 30560133), 2009 Program for New Century Excellent Talents in University (NCET), Guangxi Nature Sciences Foundation (GuiKeGong 1104003A-7), and Guangxi Health Ministry Medicine Grant (Key-Scientific Research-Grant Z201018). The present study is also partly supported by Self-raised Scientific Research Fund of the Health and Family Planning Commission of Guangxi Zhuang Autonomous Region (Z2016318), The Basic Ability Improvement Project for Middle-aged and Young Teachers in Colleges and Universities in Guangxi (2018KY0110), and Innovation Project of Guangxi Graduate Education (JGY2018037). As well as, the present study is also partly supported by Research Institute of Innovative Think-tank in Guangxi Medical University (The gene-environment interaction in hepatocarcinogenesis in Guangxi HCCs and its translational applications in the HCC prevention). We would also acknowledge the supported by the National Key Clinical Specialty Programs (General Surgery \& Oncology) and the Key Laboratory of Early Prevention \& Treatment for Regional High-IncidenceTumor (Guangxi Medical University), Ministry of Education, China. The authors thank the contributors of TCGA (https://cancergenome.nih.gov/) and UCSC Xena (http://xena.ucsc. $\mathrm{edu} /$ ) for sharing the HCC data on open access. In addition, we would like to acknowledge the helpful comments on this article received from our reviewers.

\section{Disclosure Statement}

The authors declare to have no conflict of interests.

\section{References}

1 Chen W, Zheng R, Baade PD, Zhang S, Zeng H, Bray F, Jemal A, Yu XQ He J: Cancer statistics in China, 2015 CA Cancer J Clin 2016;66:115-132.

-2 Torre LA, Bray F, Siegel RL, Ferlay J, Lortet-Tieulent J, Jemal A: Global cancer statistics, 2012 CA Cancer J Clin 2015;65:87-108.

- 3 Tang D, Nagano H, Nakamura M, Wada H, Marubashi S, Miyamoto A, Takeda Y, Umeshita K, Dono K, Monden M: Clinical and pathological features of Allen's type C classification of resected combined hepatocellular and cholangiocarcinoma: a comparative study with hepatocellular carcinoma and cholangiocellular carcinoma. J Gastrointest Surg 2006;10:987-998.

4 Farazi PA, DePinho RA: Hepatocellular carcinoma pathogenesis: from genes to environment. Nat Rev Cancer 2006;6:674-687.

5 Llovet JM, Zucman-Rossi J, Pikarsky E, Sangro B, Schwartz M, Sherman M, Gores G: Hepatocellular carcinoma. Nat Rev Dis Primers 2016;2:16018.

-6 Jiang DK, Sun J, Cao G, Liu Y, Lin D, Gao YZ, Ren WH, Long XD, Zhang H, Ma XP, Wang Z, Jiang W, Chen TY, Gao Y, Sun LD, Long JR, Huang HX, Wang D, Yu H, Zhang P, Tang LS, Peng B, Cai H, Liu TT, Zhou P, Liu F, Lin X, Tao S, Wan B, Sai-Yin HX, Qin LX, Yin J, Liu L, Wu C, Pei Y, Zhou YF, Zhai Y, Lu PX, Tan A, Zuo XB, Fan J, Chang J, Gu X, Wang NJ, Li Y, Liu YK, Zhai K, Zhang H, Hu Z, Liu J, Yi Q, Xiang Y, Shi R, Ding Q, Zheng W, Shu XO, Mo Z, Shugart YY, Zhang XJ, Zhou G, Shen H, Zheng SL, Xu J, Yu L: Genetic variants in STAT4 and HLA-DQ genes confer risk of hepatitis B virus-related hepatocellular carcinoma. Nat Genet 2013;45:72-75.

7 Roessler S, Long EL, Budhu A, Chen Y, Zhao X, Ji J, Walker R, Jia HL, Ye QH, Qin LX, Tang ZY, He P, Hunter KW, Thorgeirsson SS, Meltzer PS, Wang XW: Integrative genomic identification of genes on 8p associated with hepatocellular carcinoma progression and patient survival. Gastroenterology 2012;142:957-966 e912.

-8 Cancer Genome Atlas Research Network. Electronic address wbe, Cancer Genome Atlas Research $\mathrm{N}$ : Comprehensive and Integrative Genomic Characterization of Hepatocellular Carcinoma. Cell 2017;169:1327-1341 e1323. 


\section{Cellular Physiology Cell Physiol Biochem 2018;48:1854-1869

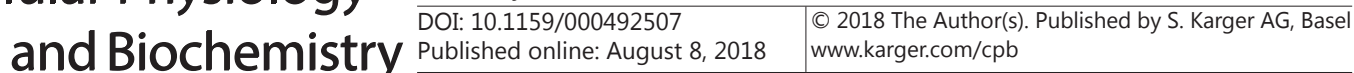

Liao et al.: Prognostic IncRNA Biomarkers in Patients with HCC

Costa FF: Non-coding RNAs, epigenetics and complexity. Gene 2008;410:9-17.

10 Mattick JS: The genetic signatures of noncoding RNAs. PLoS Genet 2009;5:e1000459.

11 Yuan JH, Yang F, Wang F, Ma JZ, Guo YJ, Tao QF, Liu F, Pan W, Wang TT, Zhou CC, Wang SB, Wang YZ, Yang Y, Yang N, Zhou WP, Yang GS, Sun SH: A long noncoding RNA activated by TGF-beta promotes the invasionmetastasis cascade in hepatocellular carcinoma. Cancer Cell 2014;25:666-681.

12 Yuan SX, Yang F, Yang Y, Tao QF, Zhang J, Huang G, Yang Y, Wang RY, Yang S, Huo XS, Zhang L, Wang F, Sun SH, Zhou WP: Long noncoding RNA associated with microvascular invasion in hepatocellular carcinoma promotes angiogenesis and serves as a predictor for hepatocellular carcinoma patients' poor recurrencefree survival after hepatectomy. Hepatology 2012;56:2231-2241.

13 Tang J, Jiang R, Deng L, Zhang X, Wang K, Sun B: Circulation long non-coding RNAs act as biomarkers for predicting tumorigenesis and metastasis in hepatocellular carcinoma. Oncotarget 2015;6:4505-4515.

14 Klingenberg M, Matsuda A, Diederichs S, Patel T: Non-coding RNA in hepatocellular carcinoma: Mechanisms, biomarkers and therapeutic targets. J Hepatol 2017;67:603-618.

15 Zheng C, Liu X, Chen L, Xu Z, Shao J: IncRNAs as prognostic molecular biomarkers in hepatocellular carcinoma: a systematic review and meta-analysis. Oncotarget 2017;8:59638-59647.

16 Cancer Genome Atlas Research N, Weinstein JN, Collisson EA, Mills GB, Shaw KR, Ozenberger BA, Ellrott K, Shmulevich I, Sander C, Stuart JM: The Cancer Genome Atlas Pan-Cancer analysis project. Nat Genet 2013;45:1113-1120.

17 Robinson MD, McCarthy DJ, Smyth GK: edgeR: a Bioconductor package for differential expression analysis of digital gene expression data. Bioinformatics 2010;26:139-140.

18 Anders S, Huber W: Differential expression analysis for sequence count data. Genome Biol 2010;11:R106.

19 Liao X, Huang K, Huang R, Liu X, Han C, Yu L, Yu T, Yang C, Wang X, Peng T: Genome-scale analysis to identify prognostic markers in patients with early-stage pancreatic ductal adenocarcinoma after pancreaticoduodenectomy. Onco Targets Ther 2017;10:4493-4506.

20 Liao X, Zhu G, Huang R, Yang C, Wang X, Huang K, Yu T, Han C, Su H, Peng T: Identification of potential prognostic microRNA biomarkers for predicting survival in patients with hepatocellular carcinoma. Cancer Manag Res 2018;10:787-803.

21 Huang R, Liao X, Li Q: Identification and validation of potential prognostic gene biomarkers for predicting survival in patients with acute myeloid leukemia. Onco Targets Ther 2017;10:5243-5254.

22 Paci P, Colombo T, Farina L: Computational analysis identifies a sponge interaction network between long non-coding RNAs and messenger RNAs in human breast cancer. BMC Syst Biol 2014;8:83.

23 Liao Q Liu C, Yuan X, Kang S, Miao R, Xiao H, Zhao G, Luo H, Bu D, Zhao H, Skogerbo G, Wu Z, Zhao Y: Largescale prediction of long non-coding RNA functions in a coding-non-coding gene co-expression network. Nucleic Acids Res 2011;39:3864-3878.

24 Dennis G, Jr., Sherman BT, Hosack DA, Yang J, Gao W, Lane HC, Lempicki RA: DAVID: Database for Annotation, Visualization, and Integrated Discovery. Genome Biol 2003;4:P3.

25 Huang da W, Sherman BT, Lempicki RA: Bioinformatics enrichment tools: paths toward the comprehensive functional analysis of large gene lists. Nucleic Acids Res 2009;37:1-13.

-26 Huang da W, Sherman BT, Lempicki RA: Systematic and integrative analysis of large gene lists using DAVID bioinformatics resources. Nat Protoc 2009;4:44-57.

27 Shannon P, Markiel A, Ozier O, Baliga NS, Wang JT, Ramage D, Amin N, Schwikowski B, Ideker T: Cytoscape: a software environment for integrated models of biomolecular interaction networks. Genome Res 2003;13:2498-2504.

28 Subramanian A, Tamayo P, Mootha VK, Mukherjee S, Ebert BL, Gillette MA, Paulovich A, Pomeroy SL, Golub TR, Lander ES, Mesirov JP: Gene set enrichment analysis: a knowledge-based approach for interpreting genome-wide expression profiles. Proc Natl Acad Sci U S A 2005;102:15545-15550.

-29 Mootha VK, Lindgren CM, Eriksson KF, Subramanian A, Sihag S, Lehar J, Puigserver P, Carlsson E, Ridderstrale M, Laurila E, Houstis N, Daly MJ, Patterson N, Mesirov JP, Golub TR, Tamayo P, Spiegelman B, Lander ES, Hirschhorn JN, Altshuler D, Groop LC: PGC-1alpha-responsive genes involved in oxidative phosphorylation are coordinately downregulated in human diabetes. Nat Genet 2003;34:267-273.

-30 Liberzon A, Birger C, Thorvaldsdottir H, Ghandi M, Mesirov JP, Tamayo P: The Molecular Signatures Database (MSigDB) hallmark gene set collection. Cell Syst 2015;1:417-425.

31 Benjamini Y, Hochberg Y: Controlling the false discovery rate: a practical and powerful approach to multiple 


\section{Cellular Physiology Cell Physiol Biochem 2018;48:1854-1869 \begin{tabular}{l|l} 
and Biochemistry 10.1159/000492507 & $\begin{array}{l}\text { () 2018 The Author(s). Published by S. Karger AG, Basel } \\
\text { www.karger.com/cpb }\end{array}$ \\
\hline
\end{tabular}

testing. J Royal Stat Soc Series B-Stat Methodol 1995;57:289-300.

-32 Reiner A, Yekutieli D, Benjamini Y: Identifying differentially expressed genes using false discovery rate controlling procedures. Bioinformatics 2003;19:368-375.

-33 Benjamini Y, Drai D, Elmer G, Kafkafi N, Golani I: Controlling the false discovery rate in behavior genetics research. Behav Brain Res 2001;125:279-284.

-34 Kersey PJ, Allen JE, Armean I, Boddu S, Bolt BJ, Carvalho-Silva D, Christensen M, Davis P, Falin LJ, Grabmueller C, Humphrey J, Kerhornou A, Khobova J, Aranganathan NK, Langridge N, Lowy E, McDowall MD, Maheswari U, Nuhn M, Ong CK, Overduin B, Paulini M, Pedro H, Perry E, Spudich G, Tapanari E, Walts B, Williams G, Tello-Ruiz M, Stein J, Wei S, Ware D, Bolser DM, Howe KL, Kulesha E, Lawson D, Maslen G, Staines DM: Ensembl Genomes 2016: more genomes, more complexity. Nucleic Acids Res 2016;44:D574580.

-35 Kersey PJ, Allen JE, Allot A, Barba M, Boddu S, Bolt BJ, Carvalho-Silva D, Christensen M, Davis P, Grabmueller C, Kumar N, Liu Z, Maurel T, Moore B, McDowall MD, Maheswari U, Naamati G, Newman V, Ong CK, Paulini M, Pedro H, Perry E, Russell M, Sparrow H, Tapanari E, Taylor K, Vullo A, Williams G, Zadissia A, Olson A, Stein J, Wei S, Tello-Ruiz M, Ware D, Luciani A, Potter S, Finn RD, Urban M, Hammond-Kosack KE, Bolser DM, De Silva N, Howe KL, Langridge N, Maslen G, Staines DM, Yates A: Ensembl Genomes 2018: an integrated omics infrastructure for non-vertebrate species. Nucleic Acids Res 2017;10.1093/nar/gkx1011

-36 He RQ, Wei QJ, Tang RX, Chen WJ, Yang X, Peng ZG, Hu XH, Ma J, Chen G: Prediction of clinical outcome and survival in soft-tissue sarcoma using a ten-lncRNA signature. Oncotarget 2017;8:80336-80347.

-37 Tang RX, Chen WJ, He RQ, Zeng JH, Liang L, Li SK, Ma J, Luo DZ, Chen G: Identification of a RNA-Seq based prognostic signature with five IncRNAs for lung squamous cell carcinoma. Oncotarget 2017;8:5076150773.

-38 Zeng JH, Liang L, He RQ, Tang RX, Cai XY, Chen JQ, Luo DZ, Chen G: Comprehensive investigation of a novel differentially expressed lncRNA expression profile signature to assess the survival of patients with colorectal adenocarcinoma. Oncotarget 2017;8:16811-16828.

39 Ma X, He Z, Li L, Yang D, Liu G: Expression profiles analysis of long non-coding RNAs identified novel lncRNA biomarkers with predictive value in outcome of cutaneous melanoma. Oncotarget 2017;8:7776177770.

40 Bao Z, Zhang W, Dong D: A potential prognostic lncRNA signature for predicting survival in patients with bladder urothelial carcinoma. Oncotarget 2017;8:10485-10497.

41 Zhong L, Lou G, Zhou X, Qin Y, Liu L, Jiang W: A six-long non-coding RNAs signature as a potential prognostic marker for survival prediction of ER-positive breast cancer patients. Oncotarget 2017;8:6786167870.

42 Shi D, Qu Q, Chang Q, Wang Y, Gui Y, Dong D: A five-long non-coding RNA signature to improve prognosis prediction of clear cell renal cell carcinoma. Oncotarget 2017;8:58699-58708.

43 Miao Y, Sui J, Xu SY, Liang GY, Pu YP, Yin LH: Comprehensive analysis of a novel four-lncRNA signature as a prognostic biomarker for human gastric cancer. Oncotarget 2017;8:75007-75024.

44 Ren W, Zhang J, Li W, Li Z, Hu S, Suo J, Ying X: A Tumor-Specific Prognostic Long Non-Coding RNA Signature in Gastric Cancer. Med Sci Monit 2016;22:3647-3657.

45 Zhang ZL, Zhao LJ, Chai L, Zhou SH, Wang F, Wei Y, Xu YP, Zhao P: Seven LncRNA-mRNA based risk score predicts the survival of head and neck squamous cell carcinoma. Sci Rep 2017;7:309.

46 Wang Z, Wu Q, Feng S, Zhao Y, Tao C: Identification of four prognostic LncRNAs for survival prediction of patients with hepatocellular carcinoma. PeerJ 2017;5:e3575.

47 Li G, Zhang H, Wan X, Yang X, Zhu C, Wang A, He L, Miao R, Chen S, Zhao H: Long noncoding RNA plays a key role in metastasis and prognosis of hepatocellular carcinoma. Biomed Res Int 2014;2014:780521.

48 Yang X, Xie X, Xiao YF, Xie R, Hu CJ, Tang B, Li BS, Yang SM: The emergence of long non-coding RNAs in the tumorigenesis of hepatocellular carcinoma. Cancer Lett 2015;360:119-124.

49 Yang Y, Chen L, Gu J, Zhang H, Yuan J, Lian Q, Lv G, Wang S, Wu Y, Yang YT, Wang D, Liu Y, Tang J, Luo G, Li Y, Hu L, Sun X, Wang D, Guo M, Xi Q, Xi J, Wang H, Zhang MQ, Lu ZJ: Recurrently deregulated IncRNAs in hepatocellular carcinoma. Nat Commun 2017;8:14421.

-50 Cui H, Zhang Y, Zhang Q, Chen W, Zhao H, Liang J: A comprehensive genome-wide analysis of long noncoding RNA expression profile in hepatocellular carcinoma. Cancer Med 2017;10.1002/cam4.1180

-51 Li CY, Liang GY, Yao WZ, Sui J, Shen X, Zhang YQ, Peng H, Hong WW, Ye YC, Zhang ZY, Zhang WH, Yin LH, $\mathrm{Pu}$ YP: Integrated analysis of long non-coding RNA competing interactions reveals the potential role in 


\section{Cellular Physiology Cell Physiol Biochem 2018;48:1854-1869

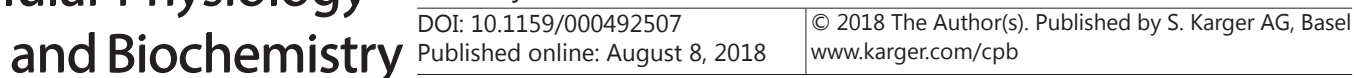

progression of human gastric cancer. Int J Oncol 2016;48:1965-1976.

52 Rong L, Zhao R, Lu J: Highly expressed long non-coding RNA FOXD2-AS1 promotes non-small cell lung cancer progression via Wnt/beta-catenin signaling. Biochem Biophys Res Commun 2017;484:586-591.

53 Yang X, Duan B, Zhou X: Long non-coding RNA FOXD2-AS1 functions as a tumor promoter in colorectal cancer by regulating EMT and Notch signaling pathway. Eur Rev Med Pharmacol Sci 2017;21:3586-3591.

54 Yu X, Lin Y, Sui W, Zou Y, Lv Z: Analysis of distinct long noncoding RNA transcriptional fingerprints in pancreatic ductal adenocarcinoma. Cancer Med 2017;6:673-680.

-55 Tu Z, He D, Deng X, Xiong M, Huang X, Li X, Hao L, Ding Q Zhang Q: An eight-long non-coding RNA signature as a candidate prognostic biomarker for lung cancer. Oncol Rep 2016;36:215-222.

56 Wu X, Ruan L, Yang Y, Mei Q: Identification of crucial regulatory relationships between long non-coding RNAs and protein-coding genes in lung squamous cell carcinoma. Mol Cell Probes 2016;30:146-152.

57 Zhang Y, Wang DL, Yan HY, Liao JY, He JH, Hu KS, Deng WX, Wang YJ, Xing HT, Koeffler HP, Yin D: Genomewide study of ER-regulated lncRNAs shows AP000439.3 may function as a key regulator of cell cycle in breast cancer. Oncol Rep 2017;38:3227-3237.

58 Xu S, Kong D, Chen Q, Ping Y, Pang D: Oncogenic long noncoding RNA landscape in breast cancer. Mol Cancer 2017;16:129.

59 Jin L, He Y, Tang S, Huang S: LncRNA GHET1 predicts poor prognosis in hepatocellular carcinoma and promotes cell proliferation by silencing KLF2. J Cell Physiol 2017;10.1002/jcp.26257

60 Li X, Su Y, Sun B, Ji W, Peng Z, Xu Y, Wu M, Su C: An Artificially Designed Interfering lncRNA Expressed by Oncolytic Adenovirus Competitively Consumes OncomiRs to Exert Antitumor Efficacy in Hepatocellular Carcinoma. Mol Cancer Ther 2016;15:1436-1451.

-61 Zhang Y, Huang JC, Cai KT, Yu XB, Chen YR, Pan WY, He ZL, Lv J, Feng ZB, Chen G: Long noncoding RNA HOTTIP promotes hepatocellular carcinoma tumorigenesis and development: A comprehensive investigation based on bioinformatics, qRTPCR and metaanalysis of 393 cases. Int J Oncol 2017;51:17051721.

62 Yang W, Li Y, Song X, Xu J, Xie J: Genome-wide analysis of long noncoding RNA and mRNA co-expression profile in intrahepatic cholangiocarcinoma tissue by RNA sequencing. Oncotarget 2017;8:26591-26599.

63 Lan X, Zhang H, Wang Z, Dong W, Sun W, Shao L, Zhang T, Zhang D: Genome-wide analysis of long noncoding RNA expression profile in papillary thyroid carcinoma. Gene 2015;569:109-117. 